\title{
Study on the Mechanism of Cancer/Testicular Antigen FSIP1 in Regulating Autophagy and Promoting Survival of Lung Cancer Cells
}

\author{
Xiaoping Zhu*, Yingping Song, Wei Wang, Weiqi Yang \\ Hexi University, Zhangye 734000, Gansu, China \\ Email:303803760@qq.com
}

\begin{abstract}
Objective - To investigate the molecular mechanism of FSIP1 gene expression and autophagy inhibition in patients with lung adenocarcinoma. Methods - TTP expression in lung adenocarcinoma H1299, H1975 and normal lung forming cells was detected by QRT-PCR and Western Bloting. Quantitative analysis and Western Bloting were performed by transfecting pCMV-FSIPI at $24 \mathrm{~h}, 48 \mathrm{~h}$ and $72 \mathrm{~h}$ respectively to analyze the expression of autophagy related factors like P62, LC3II/LCI and Beclinl. The transient transfection of overexpressed and empty plasmid was performed by adding $10 \mathrm{mg} / \mathrm{ml}$ of actinomycin D. After the termination of this transcription, RNA extraction was performed at different time points to detect the expression of Beclin1m RNA at different time points. After adding TNF-a by transfection plasmid, lung adenocarcinoma cells were divided into different groups, including no-load group, FSIPI group, no-load + TNF-A group, and FSIPI + TNF-a group. The gene expression of P50, c-Rel and NF-KBp65 in the nucleus was analyzed by immunofluorescence and Western Bloting. Lung adenocarcinoma was divided into Ikba-mut no-load group, FSIPI no-load group, 1Ikba-mut group, FSIPI group and FSIPI+ Ikba-mut group. FSIPI expression and autophagy gene expression were detected by quantitative analysis and Western Bloting. Results - In lung adenocarcinoma cells, FSIP1 RNA and protein levels were RELATIVELY low, and autophagy Becline and LC 3II/I had lower RNA and protein levels after overexpression of FSIPI compared with the no-load group. Transcription is terminated by the addition of actinomycin D. There was no significant difference in the expression of the autophagy-related gene BEC LINEM between FSIP1 and the no-load group. After overexpression of FSIPI, according to Western Bloting results, nuclear C-Rel and P65 proteins were less than those in the noload +TNF-a group and the no-load group, whileP50 protein did not change significantly. Combined with immunofluorescence studies, it was found that the expressions of c-Rel and P65 were significantly decreased after FSIP1 overexpression compared with the no-load group, while the expression of P50 was not significantly changed. Conclusion - FSIP1 is usually low expressed in lung adenocarcinoma. Overexpression of FSIP1 can effectively inhibit the nuclear metastasis of C-Rel and NF-KBp65, thus inhibiting autophagy of the cells.
\end{abstract}

Keywords: cancer/testicular antigen, FSIP1, regulation, autophagy in lung cancer cells, regulatory, mechanism

\section{Introduction}

At present, lung cancer is the main reason for the high mortality of cancer in the world, among which the rate of lung small-cell lung cancer reaches up to $85 \%$. Lung cancer has a high complication rate and mortality, and the incidence of lung cancer increases year by year, which is not conducive to people's health. In recent years, in view of the disease treatment, such treatment methods as early lung cancer screening methods and radiation therapy can improve patient survival rates. However, whether using immune therapy or targeted therapy, it is necessary to change mutated genes and molecular markers. As a result, there will be such problems as drug resistance and high cost. Based on this background, it is of great significance to look for effective molecular markers and make a detailed research on molecular mechanism. The fibrous sheath tuberculosis protein 1, also known as FSIPI, is encoded by the human HSD17B10 gene at site 15q14, so it was found in the two-hybrid protein yeast of AKAP4 in mouse. The most abundant site of the protein is in the fibrous sheath of sperm, that is to say, the coding gene is highly conserved. The expression of this gene is thought to be regulated by $\beta$-amyloid precursor proteins, whose protein products are mitochondrial enzymes that can catalyze the oxidation of various alcohols, steroids, and fatty acids, and regulate chromosome segregation to maintain the stability of the microtubule system. At the transcriptional level, most normal tissues do not express it. Due to the tissue specificity of breast cancer and bladder cancer, when FSIPI gene expression is increased, it suggests that there is a certain relationship between tumor genesis, progression and metastasis. Therefore, FSIPI is clinically used as a potential marker of malignancy. 


\section{Expression of FSIP1 in lung adenocarcinoma cells}

\subsection{Research materials and methods}

In this study, lung adenocarcinoma cell lines H1975 and H1299 and normal lung cell bead MRC-5 were selected, which were purchased from the Cell Resource Center of Shanghai Academy of Life Sciences. The reagents used in this study include PCR primers, fetal bovine serum, $0.25 \%$ trypsin, penicillin and streptomycin. The equipment used include carbon dioxide constant temperature incubator, enzyme label instrument, refrigerator, ultra-clean table, microscope, gel imager, etc. The expression level of FSIPI in lung cancer was detected by QRT-PCR, and GAPDH was used as the internal reference gene. After RNA extraction, cDNA was synthesized and amplified by PCR. In order to detect the expression of FSIPI protein in lung adenocarcinoma cells by Western blotting, it is necessary to extract the protein, and determine the concentration, and configure buffer solution, so as to detect FSIPI protein by hybridizing electrophoresis, electrotransfer and antibody.

\subsection{Research results}

The expression of MRC-5, H1299 and H1975 cells were detected by Western Product and QRT-PCR. The results showed that the protein expression of FSIPI was relatively low in H1975 and H1299, so was it under the mRNA level, as shown in the figure below.

A

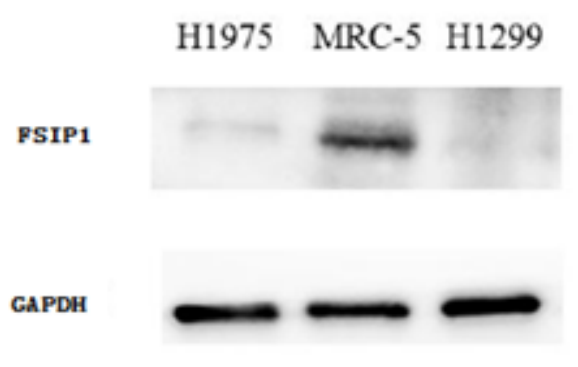

B

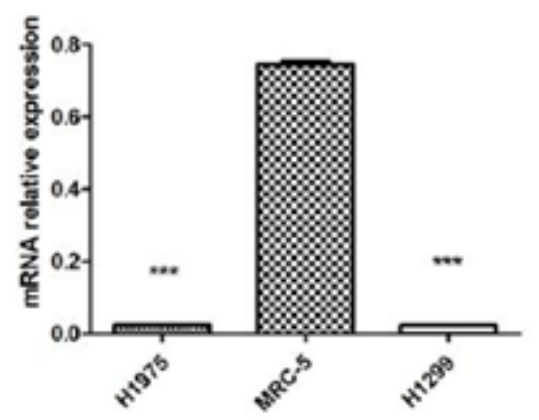

Figure 1. Research results about MRC-5, H1299 and H1975 cells

\subsection{Discussion}

As an oncosuppressor gene, FSIP1 is regulated by a variety of factors. In this study, the expression of FSIP1 in lung adenocarcinoma and human normal lung epithelial cells was detected, and the results showed that the expression of FSIP1 in lung adenocarcinoma H1975 and H 1299 cell lines was decreased compared with that in normal lung epithelial cells. To study the mechanism of FSIP1 in lung cancer, H1975 and H1299 can be selected as research materials. After the overexpression of FSIPI, the influence and mechanism of FSIPI on autophagy can be detected, which can provide theoretical basis for elucidating the role of FSIP1 in the occurrence and development of lung cancer.

\section{Effect of FSIP1 on autophagy gene in lung adenocarcinoma cells}

\subsection{Research materials and methods}

In this study, the constructed FSIPI was used to overexpress the vector, DH5A competent cell and empty plasmid. The cells used in the study were human lung adenocarcinoma cell lines H1975 and H1299, both of which had low expression of FSIP1. The reagents used were ampicillin, LB medium, LB nutrient AGAR, PCR primer, plasmid transfection agent, and actinomycin D. In the specific research process, vectors should be constructed and divided into two groups, namely, no-load control group, which transfected FSIP1 plasmids with H1299 and H1975. After transfection, cells were collected at 24h, 48h and $72 \mathrm{~h}$ respectively, and corresponding indexes were measured.

The autophagy gene expression of FSIP1 was detected by qRT-PCR and Western Bloting respectively. And the protein expressions of P62, FSIP1, Beclin1 and LC311-1 in H1975 and H1299 could also be analyzed by Western Bloting. The expression levels of FSIPI, P62, Beclin1 and LC3II/ ImRND in lung adenocarcinoma cells, H1975 and H1299 were detected by qRT-PCR.

\subsection{Research results}

After transient transfection of lung adenocarcinoma cell line H1975 and H1299 cells with FSIP1, the protein expressions of autophagy genes including LC3II/LC3I, P62 and Beclin1 were detected. The results showed that for H1975, after 48 hours 
of infection, the expression of FSIP1 protein and RNA was relatively high; and with the increasing of FSIP1 expression, the expression of Beclin1 was decreased, and the protein and RNA expressions were also significantly reduced in the no-load group, so was the ratio of LC3II to LC31. For H1299 and H1975 lung adenocarcinoma cells, P62 expression is unstable. Overexpression of FSIP1 and empty plasmid were transmitted in H1975 cells. After transfection for 48 hours, a certain amount of actinomycin D was added to prevent this transcription. Beclin1 mRNA expression level was detected at 0min, 30min, 60min, 90min and 120 min respectively. The results showed that Beclin1 mRNA expression in H1975 lung adenocarcinoma cells showed no significant difference between the overexpressed FSIP1 group and the no-loaded group after transcription inhibition, indicating that FSIP1 had no significant effect on the degradation of BEC LINL mRNA after transfection.

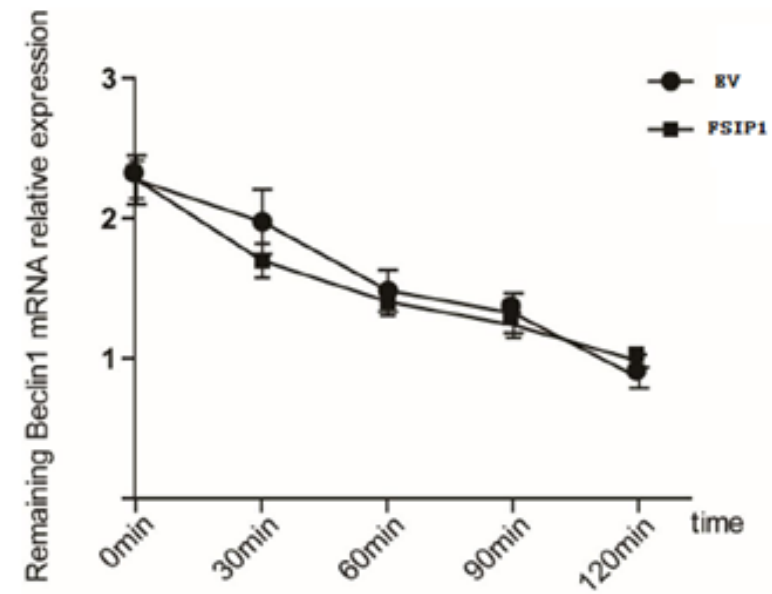

Figure 2. Remaining Beclin1 mRNA relative expression of EV and FSIP1

\subsection{Discussion}

FSIP1 is an anti-inflammatory and anti-cancer cell that can inhibit the rapid proliferation of tumor cells. Specifically, it regulates cell cycle genes and carries out transcriptional control, such as P21, E2F1 and LA TS2, which leads to cell cycle arrest. Next, it can induce apoptosis by controlling apoptosis-related genes or other molecular mechanisms, or induce or inhibit cellular autophagy by regulating autophagy genes. In combination with previous studies, it was found that increasing the expression of FSIP1 in lung adenocarcinoma cells would lead to cell death, thus resulting in the cell cycle arrest of S-phase, and effectively inhibiting the proliferation of cancer cells, while having no significant effect on cell apoptosis. By examine the expression of the autophagy by FSIP1, the srudy found that FSIP1 inhibited the expression of autophagy genes, including Beclin1. After the transcription of FSIP1, it can promote the degradation of target gene mRNA and then regulate gene expression. However, in this study, after the addition of actinomycin D to inhibit transcription, there was no significant difference in the expression of mRNA at different time points between the overexpressed FSIP1 group and the no-loaded group. FSIPI had no significant effect on Beclin1 mRNA degradation, suggesting that FSIP1 did not promote degradation at the post-transcriptional level to regulate autophagy Beclin1 expression. Autophagy will promote the development of lung cancer, thereby inhibiting autophagy to effectively reduce the proliferation of lung cancer. However, the autophagy can only be achieved by the pathway of non-caspases, which will promote lung cancer cell to die, and increase the azole, thus effectively inhibiting the development of lung cancer. There is a direct relationship between the expressions as well as pathways related to autophagy and prognosis of lung cancer. However, it remains controversial on the clinical effect of autophagy on the prognosis of patients with lung cancer.

\section{FSIP1 inhibits the NF-kB signalpathway and is involved in the regulation of autophagy in lung adenocarcinoma}

\subsection{Research materials and methods}

In this study, lung adenocarcinoma cell H1975 was selected as research obeject. And the cell and plasmosin was used to extracte kit, DAPI, and cell slides so as to transfect the cells into no-load plasmid. And then it shall transfect FSIPI overexpressing plasmid group, EV+ TNF-A group and FSIP1 + TNF-a group. After transfection with plasmid, cells were collected 48 hours later for subsequent determination. Western Bloting and QRT-PCR were used to detect the expression 
of NF-kB and autophagy genes. Specifically, the protein expressions of P65, P50 and c-Rel in the nucleus and cytoplasm of lung cancer gland cells H1975 were detected by Western Bloting. After inhibiting the NF-kB signal pathway, the protein expressions of FSIPI, P62, Beclin1, LC3II and LC3I were detected. QRT-PCR was used to detect the expression of P62, FSIPI, LC3II and LC3iRNA in H1975 after NF-kB pathway transplantation.

\subsection{Testing results}

When transiently transfecting the lung adenocarcinoma cell, the protein expressions of NF-KBp65, C-Rel and p50 in the nuclei of lung adenocarcinoma cells were detected by immunofluorescence and Western bolting after 34 hours' FSIPI transfection and 2 hours' TNF-a transfection respectively. The results showed that there were a small amount of protein expressions in the nuclei. And there is no nuclear protein in the cytoplasm. These results indicated that the cytoplasm and nuclear proteins were separated effectively. After the overexpression of FSIPI gene, the expression levels of C-Rel and NF-KBP65 in the nucleus were decreased compared with the no-load group, which was statistically significant. There was no significant change of P50 in the nucleus and cytoplasm. Cell immunofluorescence studies showed that the expressions of NF-KBp65 and c-Rel in the nucleus of the overexpressed FSIPI group were significantly decreased compared with the no-load group, while the P50 was not significantly changed. In order to verify whether FSIPI affects the autophagy of lung adenocarcinoma cells through NF-kB signal, Ikba-mut gene transmission can be carried out in H1975 to inhibit NF-kB signal. After the infection of FSIPI plasmid, the NF-kB pathway was inhibited, and then the FSIPI overexpression plasmid was transfected to observe the difference in the expression of autophagy gene in the FSIP1 + Ikba-mut group and the FSIP1 group after the inhibition of NF-kB. The results showed that in the FSIPI + Eikba-mut group, the protein and mRNA expression levels of autophagy related gene Beclin1 were significantly increased compared with the over-expressed FSIPI group, which bears statistical difference. Moreover, LC3II expression was also significantly increased. According to the findings of this study, inhibition of NF-kB signal pathway in lung adenocarcinoma cells resulted in decreased inhibition of autophagy genes Beclin1 and LC3II by FSIPI.

\subsection{Discussion}

In this study, it can be found that FSIPI played an important role in tumor genesis, progression, invasion and transfection through direct regulation of tumor gene expression. FSIPI can not only act as a transcriptional lag regulator, but also act as a transcriptional suppressor to directly or indirectly control the transcription of some factors at the transcriptional level. Chronic inflammation is a major cause of malignancy. NFkB is a metastatic factor that promotes tumor formation and can be activated in colon cancer, lung cancer and breast cancer. NF-kB is closely related to the occurrence and metastasis of lung cancer, which can be involved in drug resistance, proliferation and metastasis formation of lung cancer cells by regulating tumor-related gene transcription. In combination with this study, compared with the FSIPI overexpression group and the no-load group, the protein expressions of c-Rel and NF-kB and P65 in the nucleus of lung adenocarcinoma were decreased, while the protein expressions of P50 in the cytoplasm and nucleus were not significantly changed. According to the cellular immunofluorescence study, the cell nuclei of C-Rel and NF-KBp65 were significantly decreased in the FSIP1 overexpression group, while the P50 was not significantly changed, indicating that FSIP1 can effectively inhibit the nuclear metastasis of C-Rel and NF-KBp65 in lung adenocarcinoma cells, while it has no significant effect on P50.

In conclusion, this study revealed that FSIP1 can inhibit the autophagy of lung adenocarcinoma cells through NF$\mathrm{kB}$ signal, and effectively inhibit the occurrence and development of lung adenocarcinoma and deepen the anti-cancer mechanism of FSIPI, which can also provide theoretical reference for clinical treatment targets of lung adenocarcinoma.

\section{Acknowledgments}

This article was supported by Hexi University's 2018 R\&D Innovation and Application President's Fund Project (No. XZ2018018).

\section{References}

[1] Chen J, Wang Q, Zhang W, et al. Effect of MTRR gene on apoptosis and autophagy pathways in multi-resistant epithelial ovarian cancer. Chinese Journal of Obstetrics and Gynecology. 2016; 051(004): 285-292.

[2] Wang Z. Effect of autophagy on EGFR-TKI resistance in non-small cell lung cancer and its mechanism [PhD thesis]. Wuhan: Huazhong University of Science and Technology; 2016.

[3] Ma A, Lin Y, Li J, et al. Study on the mechanism of chloroquine in regulating autophagy in EGFR-TKI resistant lung cancer cell lines (in Chinese). International Journal of Respiration. 2018; 38(18): 1361-1366. 
[4] Yin D, Yun T, Liu Y. Research progress in autophagy regulation of epidermal growth factor receptor targeting therapy in non-small cell lung cancer (in Chinese). Academic Journal of Chinese PLA Medical School. 2018; 039(005): 441-443.

[5] Su Z. Study on the role of autophagy regulation in RBM5 inhibition of lung adenocarcinoma cell proliferation and reversal of chemotherapy resistance [PhD thesis]. Changchun: Jilin University; 2016. 